\title{
Biologic anastomosis: The first case of biologic coronary bypass surgery
}

\author{
Masashi Komeda, MD, PhD, ${ }^{\text {a,b,* }}$ Akira Marui, MD, PhD, ${ }^{\text {a, } *}$ Keiichi Tambara, MD, PhD, ${ }^{\mathrm{a}}$ \\ Masaya Yamamoto, $\mathrm{PhD},{ }^{\mathrm{c}}$ Yoshiaki Saji, MD, ${ }^{\mathrm{a}}$ Takeshi Nishina, MD, PhD, ${ }^{\mathrm{a}}$ and Yasuhiko Tabata, PhD, \\ DMSc, DPharm, ${ }^{\mathrm{c}}$ Kyoto and Aichi, Japan
}

In spite of advances in the treatment of ischemic heart disease, there exist patients who are not eligible for current revascularization procedures because of diffuse coronary lesions or too-small vessel caliber. To offer a more effective therapeutic option for these patients, we developed biologic coronary artery bypass grafting (Bio-CABG), which uses sustained release of basic fibroblast growth factor (bFGF) and an omental flap as a connector between the right gastroepiploic artery (RGEA) and ungraftable coronary arteries. ${ }^{1,2}$ Here we report the first clinical case of Bio-CABG.

\section{CLINICAL SUMMARY}

We used gelatin hydrogel sheets as a sustained release carrier for $\mathrm{bFGF}^{3} \mathrm{bFGF}$ and the gelatin hydrogel sheet were prepared as previously described. ${ }^{4}$ An aqueous solution of bFGF (250 $\mu \mathrm{g} / 3 \mathrm{~mL}$ ) was applied to freeze-dried gelatin hydrogel sheets $(15 \times 10 \mathrm{~cm})$ and then left at an ambient temperature for 1 hour to incorporate bFGF into each gelatin hydrogel sheet. The sheet slowly released bFGF for approximately 3 weeks.

Bio-CABG was performed as follows. Through an uppermedian laparotomy, the stomach and omentum were exposed, and arterial branches from the RGEA to the stomach were ligated and severed from the pylorus along the greater curvature of the stomach to the end of the RGEA. Arterial branches from the RGEA to the omentum were left intact. The diaphragm just below the left ventricular (LV) inferior wall was opened, and the omentum, including the RGEA, was passed through this opening into the pericardial sac. The bFGF-incorporated gelatin hydrogel sheet was cut into several pieces so that each sheet was appropriately applied onto the ischemic area of the heart. Subsequently, the omental flap was attached to the ischemic LV area with interrupted sutures of 6-0 Prolene (Ethicon, Inc, Somer-

\footnotetext{
From the Department of Cardiovascular Surgery, ${ }^{\text {a }}$ Kyoto University Graduate School of Medicine, Kyoto, Japan; Department of Cardiovascular Surgery, ${ }^{\mathrm{b}}$ Nagoya Heart Center, Aichi, Japan; Department of Biomaterials, ${ }^{\mathrm{c}}$ Institute for Frontier Medical Sciences, Kyoto University, Kyoto, Japan.

* M.K. and A.M. equally contributed to this work.

Received for publication May 13, 2008; accepted for publication May 26, 2008; available ahead of print Sept 19, 2008.

Address for reprints: Akira Marui, MD, PhD, Department of Cardiovascular Surgery, Kyoto University Graduate School of Medicine, 54 Shogoin-Kawahara, Sakyo,

Kyoto, 606-8507 Japan (E-mail: marui@kuhp.kyoto-u.ac.jp).

J Thorac Cardiovasc Surg 2009;138:775-7

$0022-5223 / \$ 36.00$

Copyright (c) 2009 by The American Association for Thoracic Surgery

doi:10.1016/j.jtcvs.2008.05.066
}

ville, $\mathrm{NJ}$ ) so that the bFGF sheet was totally covered by the omental flap. These procedures were easily completed without cardiopulmonary bypass. The Ethics Committee of Kyoto University approved the study protocol (protocol no. 650). We obtained written informed consent from the patients.

The first patient was a 57-year-old man with an old myocardial infarction. Coronary angiographic analysis demonstrated occlusion of the right coronary artery, stenosis of the left anterior descending coronary artery, and long segments of stenoses in the 2 distal left circumflex arteries. Although dipyridamole Thallium-21 single-photon emission computed tomographic analysis indicated moderate-to-severe hypoperfusion with partial-delay fill-in in the anterior and lateral inferior walls of the LV (Figure 1, A), there were no graftable coronary arteries in the lateral and inferior LV walls. Thus we performed off-pump CABG (left internal thoracic artery to left anterior descending coronary artery) concomitantly with Bio-CABG in the lateral to inferior wall of the LV. Six-month postoperative angiography of the RGEA revealed opacification of the native obtuse marginal branch (Figure 2). Dipyridamole Thallium-21 single-photon emission computed tomographic analysis indicated recovery of blood perfusion in the lateral and inferior walls of the $\mathrm{LV}$ in addition to the anterior LV wall (Figure 1, B). Magnetic resonance images showed improvement in wall motion, wall thickness, and systolic thickening in the lateral to inferior $\mathrm{LV}$ wall (Figure $1, C-F)$. The serum level of bFGF was within the normal range (ie, unchanged) during the whole study period.

\section{DISCUSSION}

Several experimental and clinical studies have reported promising results with therapeutic angiogenesis as an alternative to conventional medical or surgical interventions. However, they showed myocardial "staining" at best, and none of them showed opacification of the coronary arteries; arterial opacification is important because coronary flow reserve can increase not only through capillaries but also through arteries/arterioles. To the best of our knowledge, this is the first clinical report that shows macroscopic opacification of coronary branches by means of therapeutic angiogenesis. In the foregoing methods of angiogenesis, the quality of donor coronary arteries often limits the efficacy of angiogenesis because they are often diseased or stenosed. 


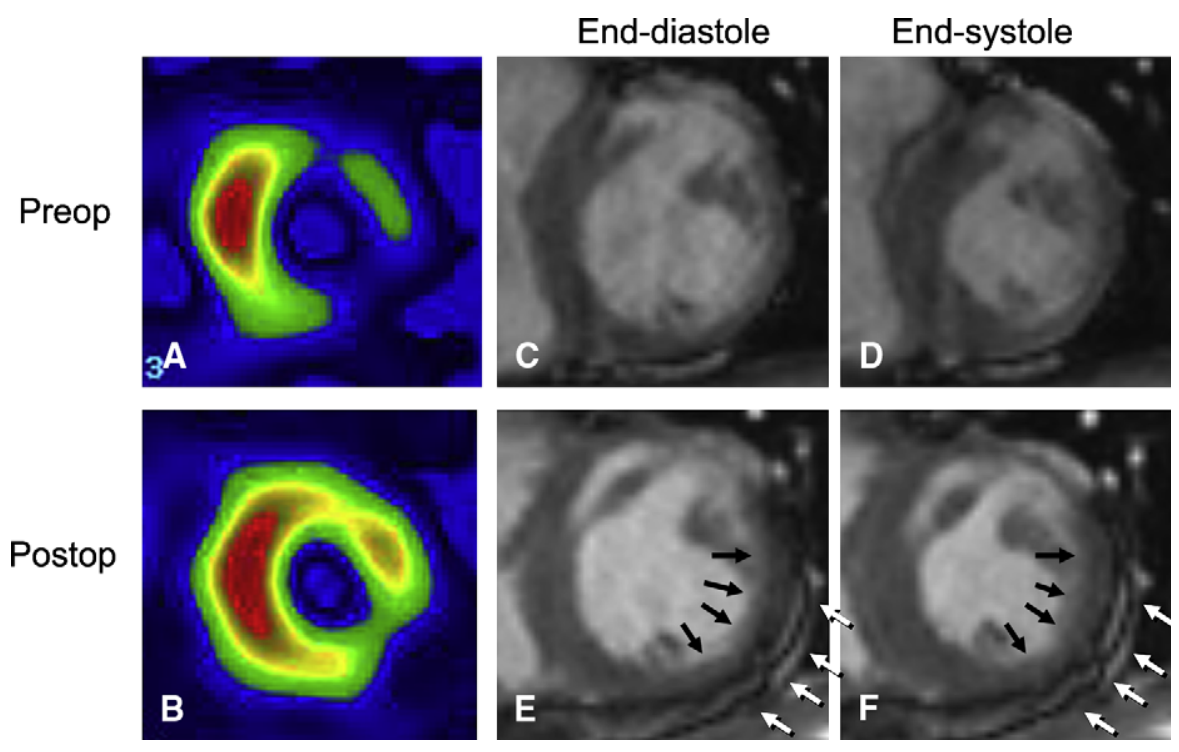

FIGURE 1. Preoperative and postoperative radiologic examinations (short-axis view). A and B, Thallium single-photon emission tomography at stress. C to F, End-diastolic and end-systolic images of cine magnetic resonance scans. Blood perfusion at stress in the lateral to inferior left ventricular wall improved after surgical intervention (A and B). Myocardial wall thickness and systolic wall thickening (E and F, black arrows) improved in the lateral to inferior left ventricular wall after surgical intervention. Preop, Before the operation (A, C, and D); Postop, after the operation (B, E, and F). White arrows, Omental flap.

In contrast, here the RGEA, which supplies blood perfusion to the omental flap, can play an important role as an independent, unobstructed, and extracardiac blood source to provide additional collateral flow (shear stress) for enhancing arteriogenic activity in the chronically ischemic region. ${ }^{1,2}$

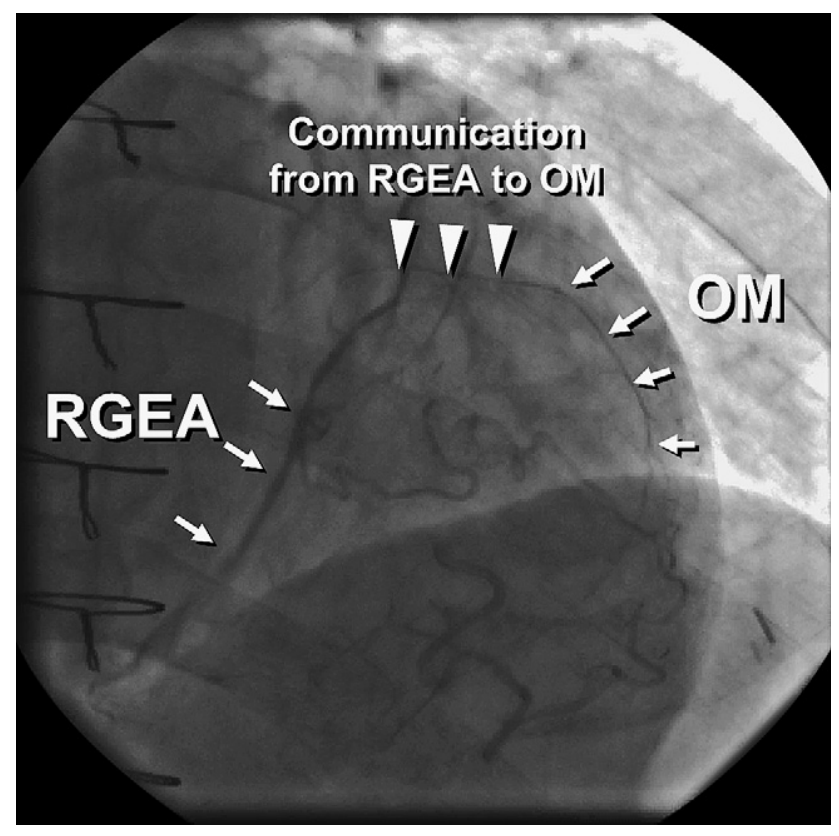

FIGURE 2. Postoperative angiography from the right gastroepiploic artery. The native obtuse marginal branch was clearly opacified from the right gastroepiploic artery. There exists a communicative vessel (angiogenesis) between the right gastroepiploic artery and the obtuse marginal branch. $R G E A$, Right gastroepiploic artery; $O M$, obtuse marginal branch.
In the present study we demonstrated the therapeutic effects of the Bio-CABG. It was noteworthy that the native coronary artery was opacified from the extracardiac blood source, which indicates that Bio-CABG connected the gastroepiploic artery to the coronary arteries without surgical anastomoses and provided additional perfusion from the RGEA to the ischemic LV wall and improved regional LV wall motion. We had shown the opacification of coronary branches by the gastroepiploic artery in animal models, ${ }^{1,2}$ but here we confirmed it for the first time in a human patient. Omentum is known to be rich in many angiogenic growth factors, including vascular endothelial growth factor, chemotactic factors, and progenitor cells. ${ }^{5}$ In addition, bFGF has synergistic/additional effects with other angiogenic agents, such as vascular endothelial growth factor, hepatocyte growth factor, or platelet-derived growth factor $\mathrm{BB} .{ }^{4}$ Thus Bio-CABG might be a promising strategy to achieve more sufficient therapeutic angiogenesis.

In conclusion, we report the first clinical case of bioanastomoses, as shown by the opacification of coronary arteries by RGEA without surgical anastomosis by Bio-CABG. BioCABG might be an alternative therapeutic method of angiogenesis in patients with severe coronary artery disease who have no other options. Further study is warranted.

\section{References}

1. Ueyama K, Bing G, Tabata Y, Ozeki M, Doi K, Nishimura K, et al. Development of biologic coronary artery bypass grafting in a rabbit model: revival of a classic concept with modern biotechnology. J Thorac Cardiovasc Surg. 2004;127:1608-15.

2. Takaba K, Jiang C, Nemoto S, Saji Y, Ikeda T, Urayama S, et al. A combination of omental flap and growth factor therapy induces arteriogenesis and increases myocardial perfusion in chronic myocardial ischemia: evolving concept of biologic coronary artery bypass grafting. J Thorac Cardiovasc Surg. 2006;132:891-9. 
3. Tabata Y, Hijikata S, Ikada Y. Enhanced vascularization and tissue granulation by basic fibroblast growth factor impregnated in gelatin hydrogels. J Control Release. 1994;31:189-99.

4. Marui A, Tabata Y, Kojima S, Yamamoto M, Tambara K, Nishina T, et al. A novel approach to therapeutic angiogenesis for patients with critical limb ischemia by sus- tained release of basic fibroblast growth factor using biodegradable gelatin hydrogel: an initial report of the phase I-IIa study. Circ J. 2007;71:1181-6.

5. Litbarg NO, Gudehithlu KP, Sethupathi P, Arruda JA, Dunea G, Singh AK. Activated omentum becomes rich in factors that promote healing and tissue regeneration. Cell Tissue Res. 2007;328:487-97.

\title{
Technical options for the treatment of anomalous origins of right or left coronary arteries associated with aortopulmonary windows
}

\author{
Bertrand Léobon, MD, Emmanuel Le Bret, MD, PhD, Régine Roussin, MD, Chokri Kortas, MD, \\ Mohammed Ly, MD, Anne Sigal-Cinqualbre, MD, Emre Belli, MD, and Alain Serraf, MD, PhD, \\ Le Plessis-Robinson, France
}

The association of an aortopulmonary window (APW) and an anomalous origin of a coronary artery is an exceptional condition with pathophysiologic specificities that make its preoperative diagnosis difficult. We report 2 new cases of APW with anomalous origin of either the right coronary artery (RCA) or the left coronary artery (LCA) and discuss surgical options for their repair.

\section{CLINICAL SUMMARY}

A 8-year-old girl $(17 \mathrm{~kg})$ was admitted for an APW revealed by a systolic murmur. Electrocardiogram showed a sinus rhythm and no ischemic sign. The chest radiograph demonstrated increased pulmonary vascularization, and the cardiothoracic ratio was 0.7 . The echocardiography demonstrated a left ventricular dilatation, low-grade mitral and aortic regurgitations, a large APW, and a severe pulmonary hypertension. The origin of the LCA was identified in a normal position, but the origin of the RCA was not seen. Under normothermic cardiopulmonary bypass, after a vertical aortotomy, the left ostium was seen in the left sinus and the right ostium was located on the pulmonary side of the APW. The pulmonary trunk was transected, and the RCA was excised with a large button of pulmonary artery wall. This defect was repaired with an autologous pericardial patch. The RCA was reimplanted in the right aortic sinus, and the APW was closed with a polytetrafluoroethylene patch (Figure 1). The postoperative course was uneventful, without

\footnotetext{
From the Department of Pediatric Cardiac Surgery, Centre Chirurgical Marie-Lannelongue, Le Plessis-Robinson, France.

Received for publication March 26, 2008; accepted for publication July 5, 2008; available ahead of print Sept 9, 2008.

Address for reprints: Alain Serraf, MD, PhD, Department of Pediatric Cardiac Surgery, Centre Chirurgical Marie-Lannelongue, 133 avenue de la Résistance, 92350 Le Plessis-Robinson, France (E-mail: a.serraf@ccml.fr).

J Thorac Cardiovasc Surg 2009;138:777-8

$0022-5223 / \$ 36.00$

Copyright (c) 2009 by The American Association for Thoracic Surgery

doi:10.1016/j.jtcvs.2008.07.013
}

ischemia on the electrocardiogram or on echocardiography. At 2 years, follow-up, the patient has a normal ventricular function with New York Heart Association class I.

A 7-month-old boy (7 kg) was admitted for an APW diagnosed on a systolic murmur and a mild dyspnea. Electrocardiogram showed no ischemic sign. On chest radiograph, the cardiothoracic ratio was 0.6 and an increased pulmonary vascularization was noted. Echocardiography demonstrated a left ventricular dilatation and a large APW with isosystemic pulmonary hypertension. No anomaly of coronary arteries was identified. Under normothermic cardiopulmonary bypass, a vertical aortotomy was performed and the LCA ostium was seen on the posterior wall of the pulmonary trunk. The repair consisted in a tunnel made of the equine pericardial patch used to close the APW, leaving the LCA ostium on the aortic side of this patch. A small polytetrafluoroethylene patch was used to close the aortotomy. The postoperative course was uneventful. The echocardiography performed the day after surgery revealed a left ventricular dysfunction and grade I mitral regurgitation. Both were regressive on echocardiography at discharge, 9 days after surgery. A postoperative computed tomography scan was performed to assess the tunnel patency (Figure 2). At 4 months follow-up, the patient is asymptomatic.

\section{DISCUSSION}

The association of an APW and anomalous origin of a coronary artery is an exceptional condition. It was first described by Stopfkuchen and colleagues ${ }^{1}$ for the RCA, by Bourlon and colleagues ${ }^{2}$ for the LCA, and by Chopra and colleagues $^{3}$ for the circumflex artery. To date, 11 RCAs, 2 LCAs, and 2 circumflex anomalous coronary arteries with APW have been reported in the literature.

Diagnosis is difficult with the lack of myocardial ischemic symptoms because of the peculiar pathophysiology encountered in this association: The abnormal coronary vessel originating from the pulmonary artery is perfused by a well- 